\title{
Improvement of the procedure of new filter masks development
}

\author{
Serhii Cheberyachko, Yurii Cheberyachko, Dmytro Radchuk, Oleg Deryugin, and Olha \\ Nesterova* \\ Dnipro University of Technology, 19, D. Yavornyts'kogo av., Dnipro, Ukraine
}

\begin{abstract}
The purpose is to substantiate theoretically the design of elastomeric half mask and canister of respirator of long-term protection. To develop the models for theoretical calculations of protective properties of dust masks the theory of non-stationary filtration and hydro aerodynamics was applied. To develop the digital model of a head the downhill simplex method was applied reflecting separate face features considering sex or age; to determine the halfmask surface the free energy equation for bowed plate being described by two-dimension spline surface was used with its unknown coefficients determined by method of proportional parts based on three-dimension coordinates of anthropometric face features. The main anthropometric face features influencing halfmasks design were determined to develop polygonous 3D model of head. According to its determined basic coordinates the surface of halfmask was determined using method of interpolation. Considering the mathematical model describing the movement of dust flows near a halfmask and in canister the dependences of distribution of air flow speed in a canister of variable geometry were set and kinematic parameters of dust-laden airflow according to a canister volume were determined. Reasonable geometric parameters of canister were determined; transient modulation from filter to inlet valve hole is performed as a contractor.
\end{abstract}

\section{Introduction}

Respiratory system protection from aerosols remains quite an urgent issue. Adequate use of high-quality one-use or reusable masks plays an important role in its solution. The efficiency of their use is directly dependent on physical and chemical properties of filter or sorption and filter materials and halfmask design to provide reliable isolation of employee's respiratory system from hazardous substances.

The process of development of individual respiratory protective devices (IRPD) may be described as a complex of research activities for search of ideas, principles, the proper calculations, and studies aimed at providing the required features and technical documents development to model and perform the new product with respect to the proper performance specification. The following five stages of the process may be distinguished: projective,

\footnotetext{
*Corresponding author: olnesterova1@rambler.ru
} 
preparatory, industrial, and post-industrial compliance assessment [1]. The projective stage consists in marketing and pre-project researches to collect the information for the preparatory stage aimed at organizational and economical production arrangement and materials preparation. IRPD designing is mainly performed by means of heuristic approaches mostly based on the developers' ultimate beliefs, the experience of previous projects, databases on known similar products. Thus, it can be described using the framework representing the main project stages (Fig. 1):

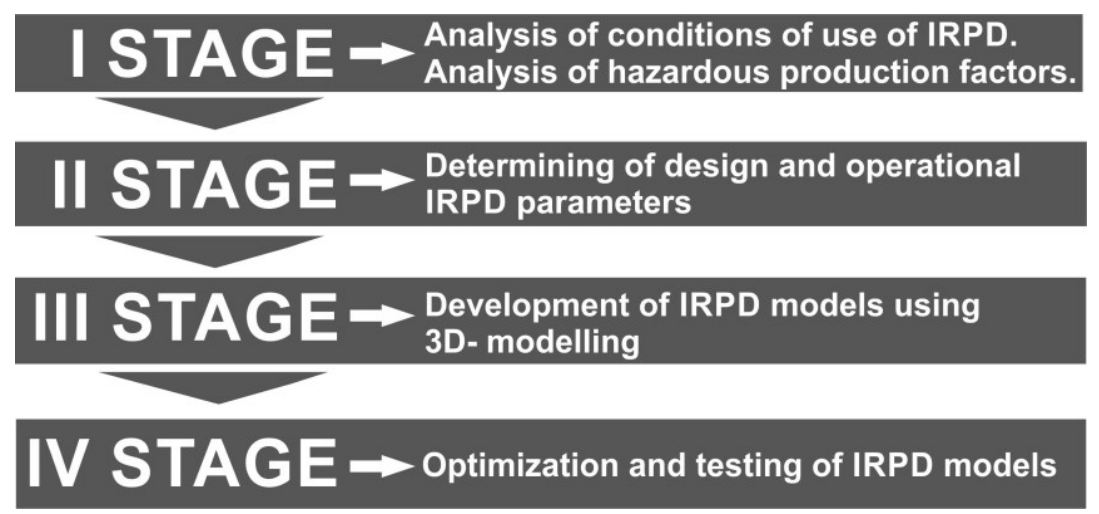

Fig. 1. The main stages of process of new IRPD development

The quality of IRPD design is determined by the performance characteristics of components meeting the requirements stated in regulatory documents. The most important of them are as follows $[2,3]$

- factors of application of filtering elements being determined by functions of IRPD: penetration factor, dust capacity;

- ergonomic factors of inhalation-exhalation valve systems, contact line, facepiece fasteners: inhalation and exhalation resistance, carbon dioxide content in the exhaled air, facepiece correspondence to anthropometric parameters of a face, field of vision, distinctness of speech;

- reliability factors (option of storage stability up to the declared expiry date): protective action period.

Filter IRPD in accordance with their purpose of function should provide purification of exhaled air from hazardous substances to the concentration not exceeding maximum permissible concentration. Table 1 provides the relevant data for choice of mask components to solve the task of employees' protection from the definite hazard. Modern filters allow sufficient protection even from submicron particles. But the canisters design does not allow using the full protective capacity of filters. This reduces the period of protective action of dust masks.

The protective properties of IRPD are mostly influenced by the occurrence of gaps between a half mask and face caused by imperfection of obturator design, which is not responsive to the anthropometric parameters of faces.

The method of components is commonly applied now. It is based on ten different parameters of users' faces to be used for calculations of complex indicators, so-called PC1 and PC2 components [4].

PC1 (first component) $=0.343264 *$ (minimum frontal breadth) $+0.426498 *$ (face width) $+0.372717^{*}$ (bigonial breadth) $+0.329648^{*}$ (face length) $+0.363474 *$ (interpupillary breadth) $+0.372241 *$ (head breadth) $+0.113578 *$ (nose protrusion) + $0.301125 *$ (nose breadth) $+0.202311 *$ (nasal root breadth $)+0.193650 *$ (subnasale-sallion length). 
PC2 $($ second component $)=-0.152951 *($ minimum frontal breadth $)-0.039087 *$ face width) - 0.093279* (bigonial breadth) $+0.359799 *$ (face length) - 0.173099* (interpupillarybreadth) $+0.013306^{*}$ (head breadth) $+0.551842 *$ (nose protrusion) 0.210833* (nose breadth). Two components are not considered in comparison to PC1.

Table 1. The main performance specifications for IRPD design development

\begin{tabular}{|c|l|}
\hline Hazards & \multicolumn{1}{|c|}{$\begin{array}{c}\text { Air disperse particles of various origin (dust, smoke, fog), } \\
\text { toxic gases, and vapours }\end{array}$} \\
\hline $\begin{array}{c}\text { Components of } \\
\text { filtering IRPD } \\
\text { providing protection } \\
\text { from hazards }\end{array}$ & $\begin{array}{l}\text { Obturators of different designs, including neck seal; filtering } \\
\text { components; inhalation-exhalation valve systems; fixing details. }\end{array}$ \\
\hline Operating principle & $\begin{array}{l}\text { Isolation of the space under the mask; filtering, including filtering } \\
\text { along the contact line, air-tightness resulting from tight-fitting of } \\
\text { inhalation-exhalation valve leaf and saddle }\end{array}$ \\
\hline Materials & $\begin{array}{l}\text { Air-tight materials (rubber, silicone, polyethylene etc.); filtering } \\
\text { materials (impregnated fibrous chemosorbents with indication of } \\
\text { wearing off for absorbing capacity, carbohydrate fibrous } \\
\text { materials, catalystsfor low-temperature oxidation, filtering } \\
\text { materials); supplementary materials. }\end{array}$ \\
\hline Control indicators & $\begin{array}{l}\text { Inward leakage: } \\
\text { - via contact line; } \\
\text { - via inhalation and exhalation valve. } \\
\text { Ratio of penetration through filtering material. } \\
\text { Effective protection time. }\end{array}$ \\
\hline
\end{tabular}

According to the components described five categories of employees' face types are distinguished: small, short - wide, medium, long and tall, large (Fig. 2). They become the base for determining the requirements to the frame of IRPD.

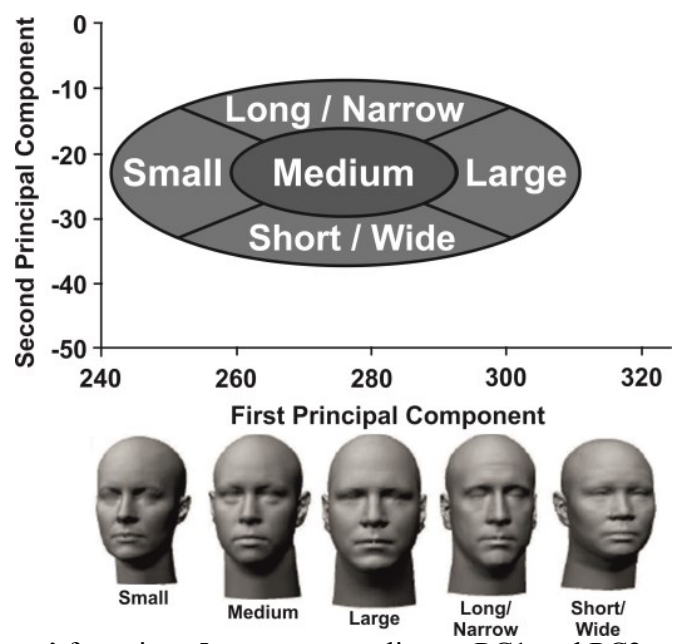

Fig. 2. Distribution of users' faces into 5 groups according to PC1 and PC2 components [4]

The approach does not provide reliable isolation of worker's respiratory organs by means of dust respirators and requires the proper studies for digital models development of half masks surfaces to correct their design according to anthropometric parameters of the 
person's face.Besides, the unification of half masks design to provide fast production with respect to their functions under the working conditions is one of the trends of product-line expansion for IRPD. This results in need of algorithm development for computer assisted design enabling fast corrections and final product prototype [5]. It is important to control the performance of different components of dust respirators continuously both at the stages of design and exploitation; this requires the development of the proper control methods of IRPD operating parameters [6]. .

\section{Improvement of procedure of development of new half mask}

The described approach to the development of IRPD is a long-term one; it may cause the situation of the developed and produced mask being outmoded and non-corresponding to rapidly changing conditions. This results in the fact that major producers widely apply modern computer technology in the development of halfmasks. In such a case the algorithm of new IRPD development includes five consistent stages:

1. 3 D scanning is often widely used for study of anthropometric parameters of worker's faces (e.g. Artec EVA 3D scanner ); the obtained digital images are processed by the applied software (e.g. Geomagic Studio v12.0 3D-системa, Rock Hill, SC), which are later used for real dimensions determining (e. g. using Active Shape Models method or method of integrated models); in order to determine correlation between the normal line $l$ and subspace $\pi$ it is needed to set parameters of camera and projector of their location (Fig. 3) from matrix equation [7]:

$$
[0,1-d(i)] \times P p[x, y, z)]^{\mathrm{T}}=0
$$

Where $P p$ stands for projection matrix of projector; $(x, y, z)$ stand for coordinated of point belonging the normal line, $T$ stands for matrix of geometric transformation.

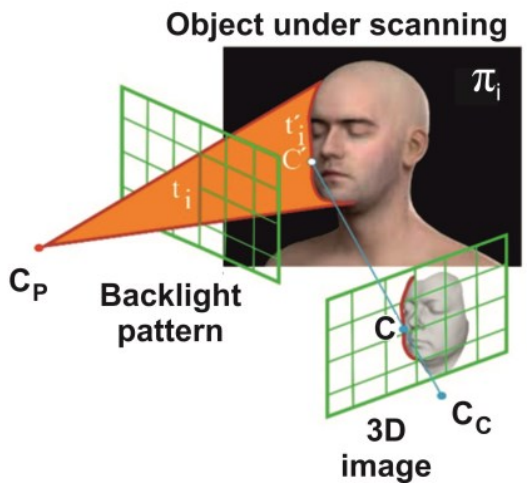

Fig. 3. Frame work of model building of optical triangulation: $C p$ and $C c$ stand for optical centre of projector and camera

2. The building of digital model of head based on polygon mesh points with key anthropometric points on the scanned images determining the obturator adumbration (in most cases they include face length and width, lips width, nose breadth and length). This was performed by means of coordinates determining of anthropometric points on the scanned faces images using software with $3 \mathrm{D}$ reconstruction, and digital image was obtained by means of weighted Gaussian function of radial type and local fitting. At the obtained points cloud of scanned head image with previously determined pixel coordinates 
we put the image draft. It is in the form of vectors $d=\left(d_{1}, d_{2}, \ldots d_{n}\right)$ with the elements being the indices of points $\mathrm{d}_{\mathrm{i}}$ of basic polygonous model [8]; vectors are determined by the polygonal curve passing the model points, each polygonal curve describing a single face feature (Fig. 4).
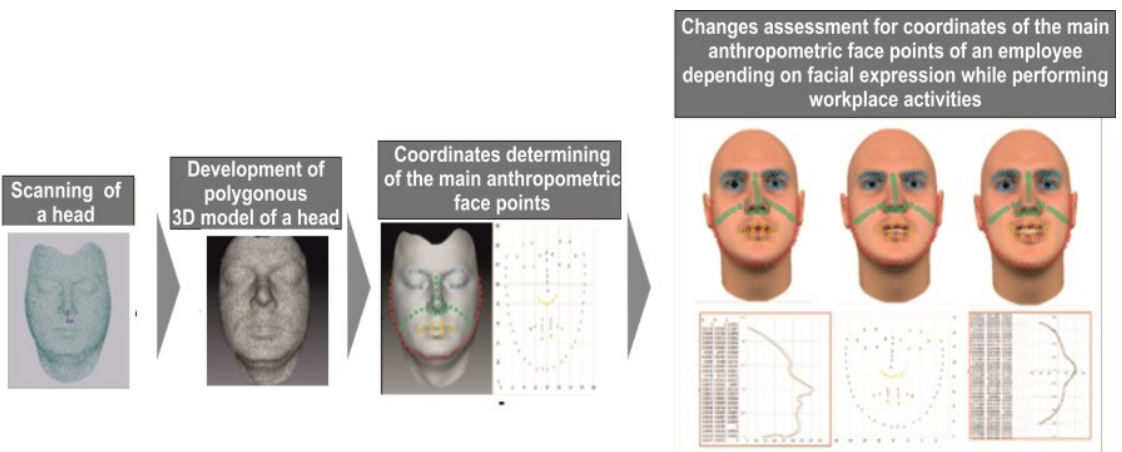

Fig. 4. Stages of determining of coordinates of anthropometric of employee's face

3. Building of half mask 3D surface by means of NURBS-surfaces, defined by subsequent multilevel transformation of shape and parameters of digital face image being split into some levels with the proper division of the function of creation process into separate subfunctions. The classic method of linear interpolation (the properpolynomial development defined in Newton form) was applied for this problem solution. The approach is based on the Weierstrass theorem stating that certain continuous over all of the intervals function may be given a reasonable approximation for polynomial.

The surface obtained may be transformed into the different models (Polygon, CADmodel, FE-model) in order to get more accurate image and level of correspondence to face parameters (Fig. 5).
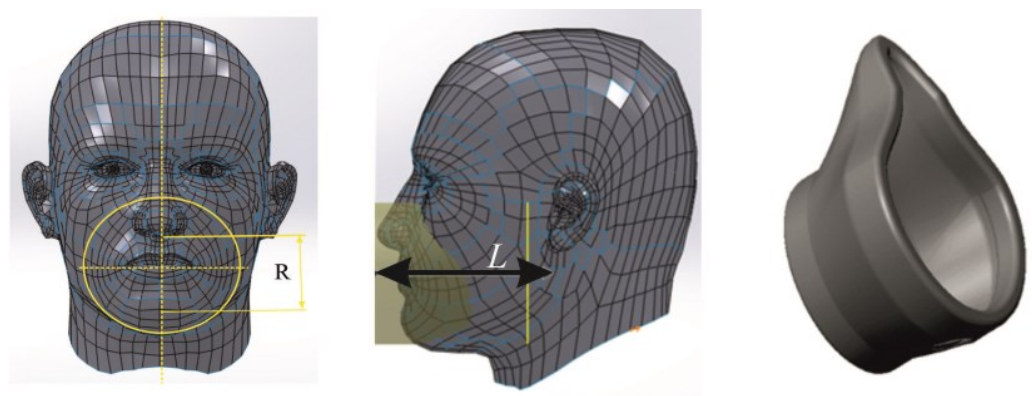

Fig. 5. Some stages of half mask surface development

4. Choosing the proper filtering materials to produce halfmask of respirator, e.g.: multifunctional chemosorbents for toxic gases and vapours capturing or filters with the determined term of protective ability in accordance with the exploitation conditions. As for filtering halfmasks it is possible to carry out stressing and stretch calculations for their frameworks to ensure the ability to hold shape during the inhalation process and change geometry according to facial anthropometric peculiarities (ANSYS software allows simulation of air flow, analysis of influence of temperature, pressure, mechanical deformation, and hold down pressure on halfmask[9]) at this stage.The elastomeric halfmasks require calculations for canisters, i.e. its diameter and outlet dimensions, as they 
influence the distribution of air flow inside the filter providing thus the determined protective ability (Fig. 6, 7).

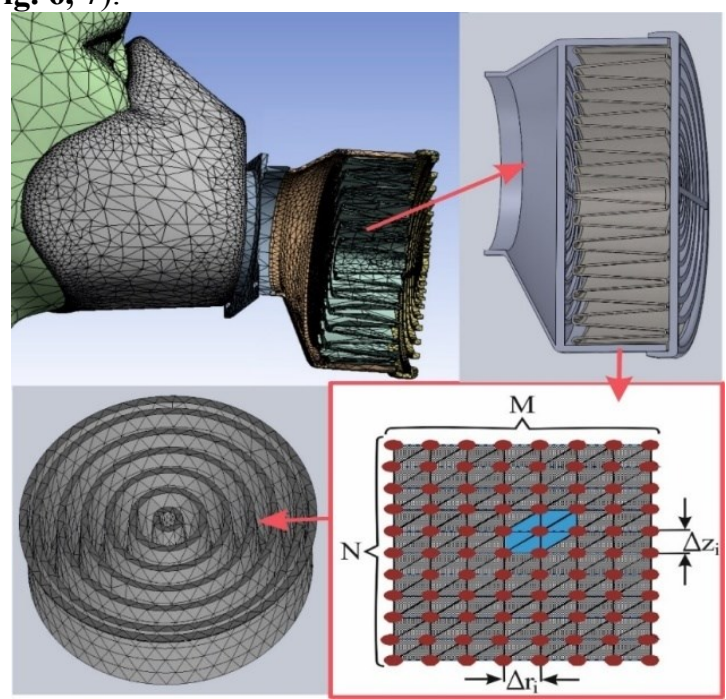

Fig. 6. Calculation pattern of half mask with canister divided into triangle end elements; $N, M$ stand for link size, $\Delta r, \Delta z$ stand for link plotting

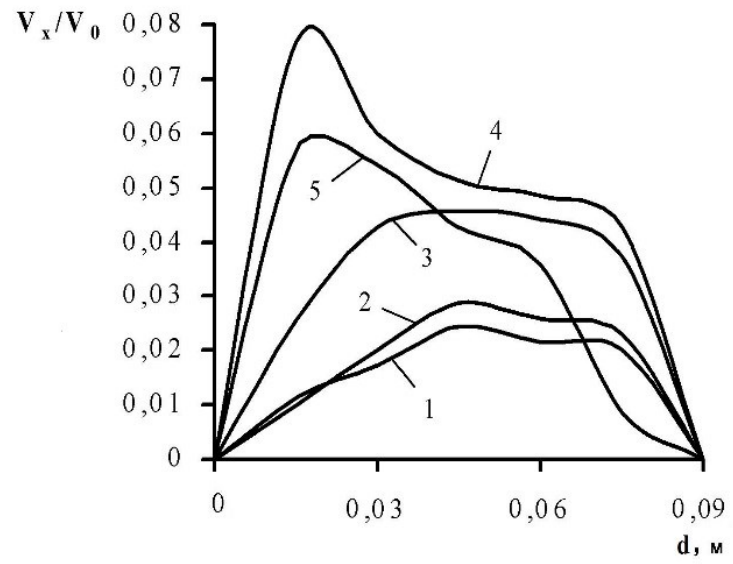

Fig. 7. Curves of relative filtration speed distribution in the canister diameter in case of $5 \mathrm{~mm}$ (1); 10 $\mathrm{mm}$ (2); $15 \mathrm{~mm}$ (3); $20 \mathrm{~mm}$ (4) shifted inlet from canister centre obtained from the data of air flows distribution using ANSYS CFX software

The suggested approach to half masks development is also different from the existing ones by the fact that each stage of transformation of scanned worker's face image into the digital image of half mask surface involves check of its correspondence to individual face parameters. The check is performed by means of comparison of three dimensional surfaces by ICP method based on minimization of distance between the points of surfaces, which are compared as the objects of vector space.

After calculations of the check of protective properties of the respirator developed its prototype may be printed using $3 \mathrm{D}$ printer. It can be used for making matrix for batch manufacturing. 


\section{Further improvement of procedure of new filtering half masks development}

As the requirements to the protection of employees are constantly rising and there exists competition between the major producers of individual respiratory protective devices, the algorithm needs constant modernization and improvement to minimize time for halfmask production and improvement of final product quality. This results in the need to consider the following issues while developing new filtering respirators:

- the peculiarities of halfmask frame development under the conditions of influence of the factors to take into account changes of anthropometric features of employees' faces, particularly with age and sex;

- parameters of production environment (microclimate, qualitative and quantitative content of hazardous production factors, peculiarities of modern materials);

- modes of operation (duration, periodicity), complexity and intensity of employees' work with minimization of additional risks resulting from electrisation and thermoplegia of workers during the process of their workplace activities;

- interrelations between the structural elements of IRPD for their integrity providing;

- optimization models of IRPD according to the criteria of protection, reliability, and ergonomics with respect to regulatory documents requirements. This enables solution of previously unsolved problems.

Analysing current trends we may point out that improvement of halfmask design algorithm will consist in the search of new efficient methods of:

- fast processing of statistical data of anthropometric parameters of faces aimed at construction of authentic digital model of head enabling consideration of employees' facial features changes with age, sex, and nationality in the digital image;

- correct development of 3D surface of halfmask to consider individual features changes for producing halfmask obturator;

- respirator quality checks both at the stage of development and at the stage of prototype production, which would enable improvement of the developed model to ensure high level of tight-fitting;

- calculation of time of protection with respect to different climate and operational parameters requiring the search of new filtering materials with the proper indication of their properties;

- theoretical estimation of protective efficiency of respirators in order to provide the assessment of protective properties of respirators with respect to their functional and operation parameters at the stage of development.

Modern approaches to IRPD allow reduction of design works and fast and high-quality development of components and means of production.

\section{Conclusions}

The analysis of IRPD development trends allows pointing out of a range of original and not realized ideas to speed-up the design process:

- development of halfmasks of filtering respirators with improved isolating properties using 3D models of employees' faces with accurate correspondence to anthropometric features;

- development of models for airflow in filtering canisters to provide uniform speed field of airflow on the whole area of filtering element in order to reduce breathing resistance and improve respiratory protection;

- equipping respirators with electronic control systems to provide the control of respiratory protection level of filtering respirators. 


\section{References}

1. M. Gutierrez, M. Galang, R. Seva, M. Lu, D. Ty IJIE 44, 131-139 (2014)

2. V. Kirillov, A. Bunchev, A. Chirkin RAMSLMIE 4, 25-31 (2013)

3. M. Cai, S. Shen, H. Li, X. Zhang, Y. Ma JOEH, 13, 50-60 (2016)

4. Y. Lin, C. Chen PLS ONE 12 (11), 1-26 (2017)

5. Z. Lei, J. Yang, Z. Zhuang JOEH 11, 227-237 (2014)

6. Al-S. Zuher, A. Belozertsev, D. Bolyasov, S. Likhachev STJ. Counter-terrorism technical devices. 16 (5-6), 132-138 (2017)

7. M. Cai, H. Li, S. Shen, Y. Wang, Q. Yang JOEH 15 (3), 226-234 (2017)

8. D. Fedorov JPIC, 1 (41), 101-104 (2013)

9. N. Vinothkumar, V. Varatharasan IJARBEST 3 (24), 198- 304 (2017) 